\title{
Perfil epidemiológico de nascidos vivos: Uma análise comparativa em um território marcada por mosaicos geográficos
}

\author{
Epidemiological profile of live births: A comparative analysis in a territory marked by geographic \\ mosaics
}

Perfil epidemiológico de los nacidos vivos: Un análisis comparativo en un territorio marcado por mosaicos geográficos

\author{
Elida Borges Lopes \\ ORCID: https://orcid.org/0000-0002-4163-3766 \\ Prefeitura Municipal de Rio das Ostras, Brasil \\ E-mail: elidaborges@id.uff.br \\ Aline Cerqueira Santos Santana da Silva \\ ORCID: https://orcid.org/0000-0002-8119-3945 \\ Universidade Federal Fluminense, Brasil \\ E-mail: alinecer2014@gmail.com \\ Alcina Frederica Nicol \\ ORCID: https://orcid.org/0000-0001-7221-5666 \\ Fundação Oswaldo Cruz, Brasil \\ E-mail: afnnicol@gmail.com \\ Giúlia Kamille de Medeiros Padilha \\ ORCID: https://orcid.org/0000-0002-9537-5731 \\ Universidade Federal Fluminense \\ E-mail: giuliapadilha@id.uff.br \\ Walesca Carvalho Amaral Batista \\ ORCID: https://orcid.org/0000-0003-3494-7481 \\ Prefeitura Municipal de Araruama, Brasil \\ E-mail: walesca_dasilva@hotmail.com \\ Virginia Maria Azevedo Oliveira Knupp \\ ORCID: https://orcid.org/0000-0001-5512-2863 \\ Universidade Federal Fluminense, Brasil \\ E-mail: virgulaknupp@yahoo.com.br
}

\begin{abstract}
Resumo
Objetivo: analisar o perfil epidemiológico dos nascidos vivos e comparar entre cinco áreas de planejamento em saúde de uma mesma região geográfica. Métodos: estudo epidemiológico, do tipo ecológico, observacional, descritivo, quantitativo, elaborado com base nos dados contidos no Sistema de Informação sobre Nascidos Vivos. Os dados foram processados nos programas Tab para Windows e analisados no LibreOffice Excel versão 5.4. Os resultados descritivos foram exibidos em frequência absoluta e relativa e os indicadores em proporções de acordo com AP de residência da mãe. Resultados: Foram analisadas 82.780 declarações de Nascidos vivos. A maioria apresentou-se a termo, o peso adequado, Apgar no primeiro e quinto minutos entre 7 a 10 pontos. As mães foram em sua maioria pardas, solteiras, com 8 a 11 anos de estudo e idade entre 20 a 34. O tipo de parto mais frequente foi à cesárea. Considerações finais: A avaliação do perfil epidemiológico dos nascidos vivos deve fazer parte dos serviços de saúde para que as ações estratégicas sejam direcionadas, com vistas a intervir nos agravos de saúde que levam a situação de prematuridade, a desigualdade do acesso ao pré-natal, e cesarianas sem critério. Logo, os serviços de saúde devem dispor de Plano de ações locais.
\end{abstract}

Palavras-chave: Nascidos vivos; Sistemas de informação em saúde; Perfil epidemiológico.

\begin{abstract}
Objective: to analyze the epidemiological profile of live births and to compare between five areas of health planning in the same geographic region. Methods: an epidemiological study, of an ecological, observational, descriptive, quantitative type, elaborated based on the data contained in the Information System on Live Births. The data were processed in the Tab for Windows programs and analyzed in LibreOffice Excel version 5.4. The descriptive results were displayed in absolute and relative frequency and the indicators in proportions according to the mother's AP of residence. Results: 82,780 live birth declarations were analyzed. Most were at term, the weight was adequate, Apgar score between 7 and 10 points in the first and fifth minutes. The mothers were mostly brown, single, with 8 to 11
\end{abstract}


years of study and aged between 20 to 34. The most frequent type of delivery was cesarean. Final considerations: The evaluation of the epidemiological profile of live births must be part of health services so that strategic actions are directed, in order to intervene in health problems that lead to prematurity, unequal access to prenatal care, and cesarean sections without criteria. Therefore, health services must have a local action plan.

Keywords: Live birth; Health information systems; Health profile.

\section{Resumen}

Objetivo: analizar el perfil epidemiológico de los nacidos vivos y comparar entre cinco áreas de planificación de la salud en una misma región geográfica. Métodos: estudio epidemiológico, de tipo ecológico, observacional, descriptivo, cuantitativo, elaborado a partir de los datos contenidos en el Sistema de Información de Nacidos Vivos. Los datos se procesaron en la pestaña de los programas de Windows y se analizaron en LibreOffice Excel versión 5.4. Los resultados descriptivos se exhibieron en frecuencia absoluta y relativa y los indicadores en proporciones según la PA de residencia de la madre. Resultados: se analizaron 82.780 declaraciones de nacidos vivos. La mayoría presentó al término, el peso adecuado, puntaje de Apgar entre 7 a 10 puntos en el primer y quinto minuto. Las madres eran mayoritariamente morenas, solteras, con 8 a 11 años de escolaridad y entre 20 y 34 años. El tipo de parto más frecuente fue la cesárea. Consideraciones finales: La evaluación del perfil epidemiológico de los nacidos vivos debe formar parte de los servicios de salud para que se orienten acciones estratégicas para intervenir en problemas de salud que conducen a la prematuridad, acceso desigual al prenatal y cesáreas sin criterio. Por lo tanto, los servicios de salud deben tener un plan de acción local.

Palabras clave: Nacimiento vivo; Sistemas de información en salud; Perfil de salud.

\section{Introdução}

A saúde materna e infantil é uma prioridade para a Organização Mundial das Nações Unidas (ONU), estima-se que a cada ano ocorram cento e trinta milhões de nascimentos no mundo e três milhões no Brasil. Ao longo dos anos foram criadas políticas, programas e diretrizes a essa população, a fim de, alcançar dois dos oito Objetivos do Desenvolvimento do Milênio, estabelecidos em setembro de 2000 para todos os países (Fundo das Nações Unidas para a Infância, 2019a; Fundo das Nações Unidas para a Infância, 2019b; Justino, Lopes, Santos \& Andrade, 2019; Rattner \& Moura, 2016)

No Brasil para melhorar o acesso, a cobertura e a qualidade da atenção pré-natal, o Ministério da Saúde (MS) lançou o Programa de Humanização no Pré-natal e Nascimento (PHPN) em 2000 e a Rede Cegonha em 2011, através da Portaria GM/MS 1.459/2011, que teve como objetivo assegurar à mulher o direito ao planejamento reprodutivo e à atenção humanizada à gravidez, ao parto e puerpério e à criança o direito ao nascimento seguro e ao crescimento e ao desenvolvimento saudável (Anjos \& Boing, 2016; Pereira \& Mudjalib, 2016).

Porém ainda havia a necessidade de reforçar os objetivos da ONU antes estabelecidos, foi criada uma nova agenda 2015-2030 com Objetivos do Desenvolvimento Sustentável (ODS), devido à persistência da mortalidade neonatal precoce. Desfecho esse que pode ser evitado por meio da análise das circunstâncias da gestação, parto e acesso aos serviços de saúde (Marques, Oliveira \& Bonfim, 2016; Organização Pan-Americana da Saúde, 2019).

A avaliação acerca das condições de nascimento é primordial para que medidas preventivas e estratégias no planejamento em saúde sejam adotadas, mediante informações encontradas no Sistema de Informações sobre Nascidos Vivos (SINASC), criado em 1990 pelo Ministério da Saúde. Nele estão descritos o peso ao nascer, sexo, índice de Apgar no primeiro e quinto minutos, idade gestacional, duração da gestação, tipo de parto, número de consultas de pré-natal, grau de instrução e idade da mãe o que torna capaz de pormenorizar o perfil epidemiológico desse segmento populacional, baseando-se nas estatísticas das características neonatais, maternas e assistenciais (Oliveira, et al., 2015; Nunes, Prudêncio, Carvalho \& Mamede, 2016).

O pré-natal é um cuidado essencial para prevenir e detectar precocemente patologias maternas e fetais, visto que promove o desenvolvimento saudável do bebê e reduz os riscos durante a gestação. Pesquisas mostram que o acompanhamento da gestação desde o primeiro trimestre e número de consultas no pré-natal decresce os índices de mortalidade infantil e materna, mediante as menores prevalências de hipertensão no período gestacional, além do aumento da cobertura de vacinação 
antitetânica e suplementação com sulfato ferroso (Faria \& Santana, 2016).

Para isso os serviços de saúde que realizam assistência ao binômio mãe-filho devem ser eficientes e resolutivos, para que mortes perinatais sejam evitadas, especialmente as ações para o pré-natal e parto, visto que esses cuidados têm impacto na redução da mortalidade neonatal precoce, além do adequado cuidado ao recém-nascido de risco que devem ser prontamente detectados pela equipe de saúde (Cardoso \& Schumacher, 2017; Pereira, et al., 2017).

À vista disso é preciso conhecer e compreender a realidade local na perspectiva da coletividade e avaliar a magnitude da assistência materno-infantil. O objetivo deste estudo é analisar o perfil epidemiológico dos nascidos vivos e comparar entre cinco áreas de planejamento em saúde de uma mesma região geográfica.

\section{Metodologia}

Estudo epidemiológico, do tipo ecológico, de investigação observacional, descritivo, com abordagem quantitativa, elaborado com base nos dados coletados no SINASC, do município do Rio de Janeiro/RJ. O referencial metodológico adotado neste estudo se insere na perspectiva quantitativa, descritiva de características coletiva que visa refletir o nível geral de cuidados à saúde, e utiliza indicadores de saúde para avaliação dos serviços de saúde (Gordis, 2010).

O município do Rio de Janeiro faz parte da Região de Saúde denominada Metropolitana I. Ele possui 153 bairros, divididos geograficamente em centro, zona sul, zona norte e zona oeste. A rede de saúde é organizada por Áreas de Planejamento (AP): AP 1.0, AP 2.0, AP 3.0, AP 4.0 e AP 5.0. Essa rede conta com 19 maternidades públicas, três para atendimento de alto risco e 16 de baixo risco.

Foram analisadas 82.780 declarações de Nascidos Vivos, com nascimento entre primeiro de janeiro a 31 de dezembro de 2016. O critério de seleção teve como base os registros com informações completa. Já o critério de exclusão diz respeito aos nascimentos que ocorreram nos anos anteriores e superiores ao de interesse.

Foi descrita variáveis neonatais: idade gestacional; peso ao nascer; anomalia congênita, índice de Apgar no primeiro minuto de vida; índice de Apgar no quinto minutos de vida. Variáveis maternas: cor/raça; estado civil; escolaridade e idade. Variáveis assistenciais: tipo de parto e consulta de pré-natal.

Os dados foram processados e analisados nos programas Tab para Windows (TabWin) e LibreOffice Excel versão 5.4. A primeira ferramenta foi utilizada para tabulação das variáveis, a segunda para análise descritiva e cálculo dos indicadores de saúde. Os resultados descritivos foram exibidos em frequência absoluta (n) e relativa (\%) e os indicadores em proporções de acordo com AP de residência da mãe.

Foram selecionados sete indicadores: (1) proporção de nascidos vivos prematuros; (2) proporção de nascidos vivos com baixo peso ao nascer; (3); proporção de nascidos vivos com Apgar < que 7 no primeiro minuto de vida; (4) proporção de nascidos vivos com Apgar < que 7 no quinto minutos de vida; (5) proporção de nascidos vivos de mães adolescentes; (6) proporção de consultas de pré-natal e (7) proporção de nascidos vivos de parto cesáreo.

Essa pesquisa faz parte do projeto de Trabalho de Conclusão de Curso de graduação em Enfermagem, vertente do projeto de pesquisa intitulado: Peregrinação das gestantes no Município do Rio de Janeiro: perfil de óbito infantil e nascimentos do grupo de pesquisa Laboratório de Estudo sobre Mulheres e Enfermagem (LEME) do Departamento de Enfermagem de Rio das Ostras da Universidade Federal Fluminense. Por isso foi submetida ao Comitê de Ética em Pesquisa (CEP) e aprovada sob parecer número 3.528.330 em cumprimento ao artigo primeiro, do seu parágrafo primeiro da resolução 510, de 7 de abril de 2016.

\section{Resultados}


Os resultados observados auxiliaram no conhecimento do perfil epidemiológico dos nascidos vivos no município do Rio de Janeiro em 2016 evidenciando que as características neonatais, maternas e assistenciais entre as AP divergem. O perfil epidemiológico dos nascidos vivos no município do Rio de Janeiro de janeiro a dezembro de 2016 foi descrito segundo AP de residência da mãe a partir das variáveis neonatais, maternas e assistenciais de 82.780 DNV conforme tabelas 1,2 e 3 .

Tabela 1 - Distribuição das variáveis neonatais dos Nascidos Vivos por Área de Planejamento (AP) no município do Rio de Janeiro/ RJ em 2016.

\begin{tabular}{|c|c|c|c|c|c|c|c|c|c|c|}
\hline \multirow[b]{2}{*}{ Variável } & \multicolumn{10}{|c|}{ Área de Planejamento } \\
\hline & \multicolumn{2}{|c|}{$\begin{array}{c}\text { AP 1.0 } \\
n=4.284\end{array}$} & \multicolumn{2}{|c|}{$\begin{array}{c}\text { AP } 2.0 \\
\mathrm{n}=8.972\end{array}$} & \multicolumn{2}{|c|}{$\begin{array}{c}\text { AP } 3.0 \\
\mathrm{n}=29.768\end{array}$} & \multicolumn{2}{|c|}{$\begin{array}{c}\text { AP } 4.0 \\
\mathrm{n}=14.193\end{array}$} & \multicolumn{2}{|c|}{$\begin{array}{c}\text { AP 5.0 } \\
\mathrm{n}=25.563\end{array}$} \\
\hline Idade Gestacional & $\mathbf{n}$ & $\%$ & $\mathbf{n}$ & $\%$ & $\mathbf{n}$ & $\%$ & $\mathbf{n}$ & $\%$ & $\mathbf{n}$ & $\%$ \\
\hline$<37$ semanas & 474 & 11 & 1047 & 11,7 & 3599 & 12 & 1746 & 12,3 & 3321 & 13 \\
\hline 37 a 41 semanas & 3737 & 87,2 & 7696 & 85,8 & 25342 & 85,1 & 12101 & 85,3 & 21427 & 83,8 \\
\hline 42 semanas ou mais & 72 & 1,7 & 229 & 2,6 & 783 & 2,6 & 338 & 2,4 & 704 & 2,8 \\
\hline Peso ao Nascer & $\mathbf{n}$ & $\%$ & $\mathbf{n}$ & $\%$ & $\mathbf{n}$ & $\%$ & $\mathbf{n}$ & $\%$ & $\mathbf{n}$ & $\%$ \\
\hline$<2500$ & 403 & 9,4 & 794 & 8,8 & 2743 & 9,2 & 1310 & 9,2 & 2422 & 9,5 \\
\hline 2500 a 3999 & 3857 & 90 & 8140 & 90,7 & 26835 & 90,1 & 12799 & 90,1 & 22951 & 90 \\
\hline 4500 ou mais & 24 & 0,6 & 38 & 0,4 & 190 & 0,6 & 83 & 0,6 & 189 & 0,7 \\
\hline Anomalia Congênita & $\mathbf{n}$ & $\%$ & $\mathbf{n}$ & $\%$ & n & $\%$ & $\mathbf{n}$ & $\%$ & $\mathbf{n}$ & $\%$ \\
\hline Sim & 50 & 1,2 & 72 & 0,8 & 307 & 1 & 119 & 0,8 & 224 & 0,9 \\
\hline Não & 4220 & 98,5 & 8839 & 98,5 & 29333 & 98,5 & 14033 & 98,9 & 25315 & 99 \\
\hline Apgar no $1^{\circ}$ minuto & $\mathbf{n}$ & $\%$ & $\mathbf{n}$ & $\%$ & $\mathbf{n}$ & $\%$ & n & $\%$ & n & $\%$ \\
\hline$<7$ pontos & 291 & 6,8 & 480 & 5,3 & 1989 & 6,7 & 819 & 5,8 & 1705 & 6,7 \\
\hline 7 a 10 pontos & 3932 & 91,8 & 8436 & 94 & 27413 & 92 & 13234 & 93,2 & 23477 & 91,8 \\
\hline Apgar no $5^{\circ}$ minutos & $\mathbf{n}$ & $\%$ & $\mathbf{n}$ & $\%$ & n & $\%$ & n & $\%$ & n & $\%$ \\
\hline$<7$ pontos & 37 & 0,9 & 98 & 1 & 330 & 1,1 & 137 & 1 & 324 & 1,3 \\
\hline 7 a 10 pontos & 4206 & 98,2 & 8843 & 98,6 & 29168 & 98 & 13950 & 98,3 & 24981 & 97,7 \\
\hline
\end{tabular}

Fonte: MS/SVS/DASIS - Sistema de Informações sobre Nascidos Vivos - SINASC. Fonte: Autores.

Tabela 2 - Distribuição das variáveis de Nascidos Vivos segundo características maternas por Área de Planejamento (AP) no município do Rio de Janeiro/ RJ em 2016.

\begin{tabular}{|c|c|c|c|c|c|c|c|c|c|c|}
\hline \multirow[b]{2}{*}{ Variável } & \multicolumn{10}{|c|}{ Área de Planejamento } \\
\hline & \multicolumn{2}{|c|}{$\begin{array}{c}\text { AP } 1.0 \\
n=4.284\end{array}$} & \multicolumn{2}{|c|}{$\begin{array}{c}\text { AP } 2.0 \\
n=8.972\end{array}$} & \multicolumn{2}{|c|}{$\begin{array}{c}\text { AP } 3.0 \\
n=29.768\end{array}$} & \multicolumn{2}{|c|}{$\begin{array}{c}\text { AP 4.0 } \\
n=14.193\end{array}$} & \multicolumn{2}{|c|}{$\begin{array}{c}\text { AP 5.0 } \\
n=25.563\end{array}$} \\
\hline Raça/cor & $\mathbf{n}$ & $\%$ & $\mathbf{n}$ & $\%$ & $\mathbf{n}$ & $\%$ & $\mathrm{n}$ & $\%$ & $\mathbf{n}$ & $\%$ \\
\hline Pardas & 2131 & 49,7 & 2312 & 25,8 & 15958 & 53,6 & 7018 & 49,4 & 15949 & 62 \\
\hline Pretas & 575 & 13,4 & 662 & 7,4 & 3368 & 11,3 & 703 & 5 & 2461 & 9,6 \\
\hline Brancas & 1538 & 35,9 & 5257 & 63,8 & 9803 & 32,9 & 6305 & 44,4 & 6928 & 27,1 \\
\hline Amarelas & 13 & 0,3 & 48 & 0,5 & 84 & 0,3 & 28 & 0,2 & 40 & 0,2 Í \\
\hline Indígenas & 3 & 0,07 & 6 & 0,07 & 19 & 0,06 & 1 & 0,007 & 20 & 0,08 \\
\hline Estado Civil & $\mathbf{n}$ & $\%$ & $\mathbf{n}$ & $\%$ & $\mathbf{n}$ & $\%$ & $\mathbf{n}$ & $\%$ & $\mathbf{n}$ & $\%$ \\
\hline Solteiras & 3252 & 75,9 & 4374 & 48,8 & 19985 & 67,1 & 8380 & 59 & 17622 & 68 \\
\hline Casadas & 857 & 20 & 3785 & 42,2 & 7886 & 26,5 & 4764 & 33,6 & 6972 & 27,3 \\
\hline Viúvas & 4 & 0,09 & 17 & 0,2 & 34 & 0,1 & 11 & 0,08 & 36 & 0,1 \\
\hline Divorciadas & 41 & 1 & 158 & 1,8 & 371 & 1,2 & 206 & 1,5 & 27 & 1 \\
\hline União estável & 110 & 2,6 & 547 & 6 & 1307 & 4,4 & 717 & 5 & 573 & 2,2 \\
\hline Escolaridade & n & $\%$ & $\mathrm{n}$ & $\%$ & n & $\%$ & $\mathrm{n}$ & $\%$ & n & $\%$ \\
\hline Sem estudo & 12 & 0,3 & 11 & 0,1 & 48 & 0,2 & 11 & 0,08 & 21 & 0,08 \\
\hline 1 a 3 anos & 73 & 1,7 & 155 & 0,8 & 460 & 1,5 & 276 & 1,9 & 353 & 1,4 \\
\hline 4 a 7 anos & 731 & 17 & 1030 & 11,5 & 4489 & 15 & 2681 & 18,9 & 4275 & 16,7 \\
\hline 8 a11 anos & 2608 & 60,9 & 2724 & 30,4 & 17615 & 59,2 & 5875 & 41,4 & 16660 & 65,2 \\
\hline 12 ou mais & 834 & 19,5 & 4932 & 55 & 6329 & 21,2 & 5055 & 35,6 & 4156 & 16,3 \\
\hline Idade & $\mathrm{n}$ & $\%$ & $\mathbf{n}$ & $\%$ & $\mathbf{n}$ & $\%$ & $\mathbf{n}$ & $\%$ & $\mathbf{n}$ & $\%$ \\
\hline 10 a 19 & 726 & 16,9 & 754 & 8,4 & 4874 & 16,4 & 1657 & 11,7 & 4253 & 16,6 \\
\hline 20 a 34 & 2848 & 66,5 & 5404 & 60,2 & 20188 & 67,8 & 9514 & 67 & 17625 & 68,9 \\
\hline 35 ou mais & 710 & 16,6 & 2814 & 31,4 & 4706 & 15,8 & 3022 & 21,3 & 3609 & 14,1 \\
\hline
\end{tabular}

Fonte: MS/SVS/DASIS - Sistema de Informações sobre Nascidos Vivos - SINASC. Fonte: Autores. 
Tabela 3 - Distribuição das variáveis de Nascidos Vivos segundo características assistenciais por Área de Planejamento (AP), no município do Rio de Janeiro/ RJ em 2016.

\begin{tabular}{|c|c|c|c|c|c|c|c|c|c|c|}
\hline \multirow[b]{2}{*}{ Variável } & \multicolumn{10}{|c|}{ Área de Planejamento } \\
\hline & \multicolumn{2}{|c|}{$\begin{array}{c}\text { AP 1.0 } \\
n=4.284\end{array}$} & \multicolumn{2}{|c|}{$\begin{array}{c}\text { AP 2.0 } \\
n=8.972\end{array}$} & \multicolumn{2}{|c|}{$\begin{array}{c}\text { AP } 3.0 \\
n=29.768\end{array}$} & \multicolumn{2}{|c|}{$\begin{array}{c}\text { AP } 4.0 \\
\mathrm{n}=14.193\end{array}$} & \multicolumn{2}{|c|}{$\begin{array}{c}\text { AP 5.0 } \\
\mathrm{n}=25.563\end{array}$} \\
\hline Consulta de pré-natal & $\mathbf{n}$ & $\%$ & $\mathbf{n}$ & $\%$ & $\mathbf{n}$ & $\%$ & $\mathbf{n}$ & $\%$ & $\mathbf{n}$ & $\%$ \\
\hline Nenhuma & 59 & 1,4 & 36 & 0,4 & 453 & 1,5 & 173 & 1,2 & 506 & 2 \\
\hline De 1 a 6 & 914 & 21,3 & 954 & 10,7 & 7103 & 23,9 & 11093 & 10,6 & 19730 & 20,3 \\
\hline 7 ou mais & 3234 & 75,5 & 7482 & 83,4 & 21782 & 73,2 & 11093 & 78,2 & 19730 & 77,2 \\
\hline Tipo de parto & $\mathbf{n}$ & $\%$ & $\mathbf{n}$ & $\%$ & $\mathbf{n}$ & $\%$ & $\mathbf{n}$ & $\%$ & $\mathbf{n}$ & $\%$ \\
\hline Vaginal & 2290 & 53,5 & 3339 & 37,2 & 13983 & 47 & 5850 & 41,2 & 11638 & 45,5 \\
\hline Cesáreo & 1994 & 46,5 & 5633 & 62,8 & 15781 & 53 & 8343 & 58,8 & 13920 & 54,5 \\
\hline
\end{tabular}

Fonte: MS/SVS/DASIS - Sistema de Informações sobre Nascidos Vivos - SINASC. Fonte: Autores.

Quanto às características neonatais, observou-se maior número de crianças a termo 75,7\% (n=70 303), peso adequado em 80,3\% ( $n=74$ 582), sem anomalia congênita 98,7\% ( $\mathrm{n}=81$ 740), Apgar no primeiro minuto entre 7 a 10 em $92,3 \%$ ( $\mathrm{n}=76$ 492) e Apgar no quinto minutos de vida de 7 a 10 pontos em 98\% (n=81.148). Em relação às características maternas, observou-se que a maioria das mulheres é parda 52,4\% ( $\mathrm{n}=43.368)$, solteira $64,8 \%(\mathrm{n}=53.613)$ com 8 a 11 anos de estudo $54,9 \%(\mathrm{n}=45.482)$ e idade entre 20 a 34 anos 67,1\% (n=55.579). Quando analisado espacialmente, percebeu-se que diferentemente das demais áreas, a AP 2.0 mostrou-se o maior percentual de mulheres brancas, com 12 ou mais anos de estudo, casadas e gestantes com 35 anos ou mais conforme tabela 2. Em relação às características assistenciais observou-se que 76,4\% ( $n=63321$ ) mulheres foram em sete ou mais consultas de pré-natal e 55,1\% $(n=45.671)$ foram submetidas às cesarianas.

$\mathrm{Na}$ análise dos nascidos vivos por meio dos indicadores selecionados para o município do Rio de Janeiro em 2016 , segundo AP de residência da mãe, verificou-se que a AP 5.0 apresentou maiores proporções de NV prematuros $13 \%$ $(\mathrm{n}=3.321)$, baixo peso ao nascer 9,5\% $(\mathrm{n}=2.422)$ e índice de Apgar menor que sete no quinto minuto de vida $1,3 \%(\mathrm{n}=324)$. Com relação a mães adolescentes maiores proporções entre as AP: 1.0, 3.0 e 5.0 conforme tabela 4.

Tabela 4 - Indicadores de Saúde Materno-infantil, segundo AP de residência da mãe no município do Rio de Janeiro, RJ, Brasil. 2016.

\begin{tabular}{|c|c|c|c|c|c|}
\hline Indicadores & AP 1.0 & AP 2.0 & AP 3.0 & AP 4.0 & AP 5.0 \\
\hline Proporção de nascidos vivos prematuros & 11 & 11.7 & 12 & 12.3 & 13 \\
\hline Proporção de nascidos vivos com baixo peso ao nascer & 9.4 & 8.8 & 9.2 & 9.2 & 9.5 \\
\hline $\begin{array}{l}\text { Proporção de nascidos vivos com Apgar < que } 7 \text { no primeiro } \\
\text { minuto de vida }\end{array}$ & 6.8 & 5.3 & 6.7 & 5.8 & 6.7 \\
\hline $\begin{array}{l}\text { Proporção de nascidos vivos com Apgar < que } 7 \text { no quinto } \\
\text { minutos de vida }\end{array}$ & 0.9 & 1 & 1.1 & 1 & 1.3 \\
\hline Proporção de nascidos vivos de mães adolescentes. & 16.9 & 8.4 & 16.4 & 11.7 & 16.6 \\
\hline Proporção de nascidos vivos de parto cesáreo & 46.5 & 62.8 & 53 & 58.8 & 54.5 \\
\hline
\end{tabular}

Fonte: MS/SVS/DASIS - Sistema de Informações sobre Nascidos Vivos - SINASC. Fonte: Autores.

Acerca da consulta de pré-natal evidencia-se o maior percentual de mães que não foram a nenhuma consulta de prénatal na área $5.02 \%(n=560)$, já o percentual de mães que foram entre uma a seis foi na AP 3.0 23,9\% (n=7.103) e a proporção daquelas que foram a sete ou mais esteve maior na AP 2.0 83,4\% (n=7.482). Nota-se que as cesarianas foram as mais realizadas em quatro AP, já os partos vaginais foram em sua maior proporção na AP 1.0 53,5\%. $(\mathrm{n}=2.290)$. 


\section{Discussão}

O perfil epidemiológico dos Nascidos Vivos possibilita identificar os problemas que acometem a saúde da população materno-infantil. Essa análise da situação de saúde corrobora em criação de planejamento, estratégias e políticas públicas de saúde para intervir nos problemas identificados. No âmbito do município do Rio de Janeiro o perfil epidemiológico dos Nascidos Vivos mostrou-se similar ao encontrado em outras pesquisas. Houve o predomínio de bebês a termo, peso ao nascer adequado, valores de Apgar no primeiro e quinto minutos entre 7 a 10 pontos, sem anomalia congênita, filhos de mães solteiras, com idade entre 20 a 34 anos e com 8 a 11 anos de estudo (Alencar, et al., 2020; Ferreira, et al., 2019).

Os aspectos do ambiente e/ou local de moradia da mãe, bem como o Índice de Desenvolvimento Humano Municipal (IDH), podem influenciar na duração da gestação e peso, culminado em neonatos prematuros e/ou de baixo peso ao nascer. Desse modo a baixa escolaridade, baixa renda, moradia inadequada e alugada são fatores de risco para prematuridade, mediante a dificuldade do acesso às informações de saúde, prestação de um cuidado efetivo ao NV e ausência proteção quando em casa inadequada (Pedraza \& Cristóvão, 2015; Teixeira, Carvalho, Rocha, Pereira \& Enders, 2018).

No município do Rio de Janeiro a situação de prematuridade acometeu 12, 3\% $(\mathrm{n}=10.187)$ de NV, percebeu-se que essa situação ocorreu acima do recomendado pela Organização Mundial de Saúde (OMS), que deve ser em torno de 11\%. Essa condição de nascimento pode estar associada aos fatores sociais, biológicos e relacionados aos serviços de saúde, a saber, o baixo nível socioeconômico e educacional, o aumento na proporção de nascimentos entre as mulheres com idade mais avançada e a crescente da cesárea eletiva (Freitas \& Araújo, 2015).

Um estudo de perfil de puérperas, realizado em uma maternidade no Vale do Rio Pardo, interior do Rio Grande do Sul, que também identificou índices elevados de nascimentos prematuros e baixo peso ao nascer, ressaltou a importância de uma equipe preparada, além da disponibilidade de estrutura mínima necessária para atender essa população (Renner, et al., 2015).

Dessa maneira, a prematuridade e o baixo peso ao nascer são condições que exigem observação devido aos riscos de sequelas e morte precoce. Essas características neonatais podem ser reduzidas com intervenções no pré-natal, por meio dos grupos de gestantes e visitas domiciliares que impactaram na redução dessas características, sendo também efetiva na adesão ao aleitamento materno exclusivo (Teixeira, et al., 2016; Silva, Lima, \& Osório, 2016).

Uma pesquisa realizada com gestantes de alto risco em um ambulatório no município de Maringá/Paraná identificou que o baixo peso ao nascer está fortemente associado à realização de cesarianas e filhos de mães com idade igual ou superior a 35 anos (Almeida, et al., 2018). Contudo, no presente estudo percebe-se que a menor proporção de bebês com baixo peso foi na área que apresentou maiores proporções de cesarianas e mães nessa faixa etária. Isto pode ser em função de uma melhor nutrição durante a gestação, visto que nessa localidade vivem pessoas com melhores rendas e maior nível de escolaridade.

Em relação à prevalência de anomalia congênita percebe-se que no município do Rio de Janeiro mostrou-se baixa. Esse dado foi semelhante ao de um estudo epidemiológico do tipo transversal que também utilizou dados secundários, realizado no município de Salvador/Bahia em 2017. Ele apontou que os casos de anomalia ocorreram com mais frequência em filhos de mães menores de 18 anos e maiores de 35 anos (Pinto Junior, et al., 2017).

Sobre o índice de Apgar (realizado para avaliar a presença de asfixia neonatal) evidenciou-se que a maioria dos NV no cenário do presente estudo teve pontuação maior que sete, ou seja, não apresentaram asfixia neonatal. Essa escala criada por Virgínia Apgar encontra-se entre as avaliações clínicas que se realiza no recém-nascido, com essa avaliação é possível saber a adaptação extrauterina do recém-nascido. Aquele que obter pontuação baixa demandará atenção especializada, uma vez que, o Apgar é um marcador importante, considerado de gravidade quando o valor estiver menor que sete (Muniz, et al., 2016; Costa, Gomes Junior \& Magluta, 2018). Nesse sentido, observa-se que a maioria dos NV no presente estudo apresentou boa adaptação fora do útero, com pouca variação entre as AP. 
Quanto às características maternas percebe-se que a idade é um fator em que pode levar as mães a terem filhos prematuros e/ou com baixo peso. Por isso os extremos de idade, isto é, gestante menor de 20 anos e acima de 35 anos devem fazer parte da avaliação de saúde na consulta de pré-natal, uma vez que, mães adolescentes ou tardias podem evoluir para desfechos desfavoráveis na gestação e parto. A adolescência tem classificação diferente do ponto de vista legal e de saúde, para o Estatuto da Criança e do Adolescente (ECA), adolescente é todo indivíduo com faixa etária entre 12 a 18 anos, já para a Organização Mundial da Saúde (OMS) é aquele com idade entre 10 a 19 anos (Vieira, et al., 2017).

Em 2016 na cidade do Rio de Janeiro três AP apresentaram maiores proporções de mães adolescentes, essa condição de gravidez precoce pode acontecer por diversos fatores entre eles: iniciação sexual antes dos 15 anos, a baixa renda e falta de diálogo, escolaridade e informação dos métodos contraceptivos ou a utilização deles de modo inadequado. Tendo em vista a iniciação precoce da atividade sexual faz-se necessário, oficinas de educação sexual nas escolas para sensibilizar os jovens sobre medidas de prevenção de gravidez nessa faixa etária (Vieira, et al., 2017; Bulhoes, Alves, Moreno, Silva, \& Dutra, 2017).

Para o Ministério da Saúde (MS) a gestação avançada ou tardia é aquela que ocorre em mulheres acima de 35 anos, considerada um fator de risco pela suscetibilidade de complicações durante a gravidez, sendo assim classificada como gestação de alto risco (Aldrighi, Wall, Souza \& Cancela, 2016). No presente estudo a distribuição espacial de mães nessa faixa etária mostrou-se com bastante variação, tendo maiores proporções nas AP 2.0 e 4.0, esse fato pode ser explicado por diversos motivos como: desejo da mulher em investir na formação e carreira profissional e pela postergação do casamento, além da grande e diversificada disponibilidade de métodos contraceptivos e avanços na tecnologia da reprodução (Alves, Feitosa, Mendes \& Caminha, 2017).

Em relação ao pré-natal sabe-se que ele se configura como um importante indicador de qualidade dos serviços de saúde que tem como objetivo garantir o desenvolvimento da gestação, para diminuir as principais causas de óbitos maternos e neonatais, mediante detecção e intervenção precoce de situações de riscos (Timm, Rodrigues, Valverde \& Ribeiro, 2019). No município do Rio de Janeiro esse indicador revelou-se com maiores tendências de acesso ou adesão ao pré-natal entre as AP 2.0, 4.0 e 5.0, já entre as AP 1.0 e 3.0 ocorreu menor número de consultas. Nessas localidades onde o acesso apresentou-se de maneira desigual há necessidade de políticas públicas assistenciais e preventivas que reforcem a importância do pré-natal na prevenção de mortalidade materna e infantil.

A adesão ao pré-natal e o número de consultas podem estar associados a fatores como nível de escolaridade materna, idade e estado civil. Estudo mostra maiores prevalências de sete ou mais consultas de pré-natal entre as mulheres acima de 30 anos, com 12 anos de estudo e casadas (Anjos \& Boing, 2016). Essa realidade também foi observada nas AP 2.0 e 4.0 dessa pesquisa, essa condição estar relacionada ao local de residência e renda, visto que nessas localidades concentram-se os bairros com melhores índices de Desenvolvimento Humano (IDH).

O pré-natal tem impacto importante na redução da mortalidade infantil, isso pode ser explicado por diversos fatores. Estratégias como captação precoce para consulta de pré-natal e busca ativa de mulheres faltosas melhoram os resultados, contribuindo no direcionamento de ações de assistência materno-infantil (Faria \& Santana, 2016; Maia, Lima, Leite, Sousa \& Primo, 2017).

No que concerne o tipo de parto, sabemos que apesar de o Ministério da Saúde ter reformulado a assistência ao parto e nascimento, de modo a diminuir as cesarianas, elas ainda representam os partos mais frequentes em todo território nacional. A prevalência dessa intervenção apresenta-se acima do recomendado pela OMS em todas as faixas etárias, independentemente do nível de escolaridade. Porém, torna-se mais frequente em mulheres com 30 anos ou mais e com 12 ou mais anos de estudo. No cenário do Rio de Janeiro esses resultados são semelhantes, pois esse parto foi o mais realizado, sendo a sua maior 
proporção na AP 2.0, onde também havia mulheres com as mesmas características sociodemográficas supracitadas (Rattner \& Moura, 2016).

A Organização Mundial da Saúde (OMS) aponta que a cesariana eleva o risco de morbidade e mortalidade materna e perinatal, e que no Brasil as taxas de cesarianas encontram-se entre as mais altas do mundo. Para a gestante e sua família a cesariana está associada ao acesso médico diferenciado, ausência de asfixia ao nascimento e sexualidade preservada (Silva, 2016).

A cesárea eletiva é uma intervenção que pode ser efetiva quando se tem justificativa clínica, contribuindo na diminuição da morbidade e mortalidade materno-infantil, haja vista o percentual permitido pela OMS. Entretanto, 90\% das cesarianas não apresentam justificadas adequadas, corroborando como principal fator para o percentual elevado de recémnascido de baixo peso (Madeiro, Rufino \& Santos, 2017).

A escolha pelo parto cesáreo é motivada por diversos contextos, como às questões sociais, econômica e cultural, A dor do parto vaginal, a preocupação com a estética, o mito que o parto vaginal é arriscado para o bebê e que a cesárea mantém intacta a anatomia do períneo e vagina são motivos que as levam escolher essa via de parto, ignorando os benefícios e as medidas farmacológicas e não farmacológicas utilizadas no parto vaginal (Almeida, Almeida, Menezes, Pinto \& Lobo, 2020). No presente estudo foi visto que a cesariana ainda se encontra entre os partos mais realizados em quatro áreas de planejamento, isto pode estar associado ao número elevado de partos de alto risco, saúde da gestante, disponibilidade de recursos tecnológicos e influência do modelo obstétrico adotado.

O monitoramento dos indicadores desse segmento populacional permite dimensionar se houve avanço, estabilização ou retrocesso nas questões sociais e econômicas de um território, já que demograficamente esse grupo é considerado o principal componente no Brasil. Nesse sentindo, constatou-se no presente estudo que as ações voltadas a essa população ainda necessita de esforços, posto que os valores das proporções de prematuridade, pontuação de Apgar no primeiro minuto e cesarianas ficaram acima dos valores desejáveis. A OMS aponta que os partos prematuros devem estar abaixo ou igual a 11\%, o Apgar no primeiro minuto abaixo ou igual a 3\% e cesariana igual ou abaixo de 15\% (Rio de Janeiro, 2017).

Um estudo realizado em uma maternidade pública de Santa Maria, Rio Grande do Sul apontou que as cesarianas estão aquém do indicado e enfatiza que ainda há cesarianas sem justificativa clínica, o que pode culminar com maus desfechos para mãe e filho (Barrios, et al., 2021).

Uma revisão integrativa que fez análise e monitoramento de taxas de cesarianas no Brasil concluiu que os dados epidemiológicos encontrados no SINASC têm potencial para formulação de indicadores e suporte aos gestores estaduais e municipais no planejamento de ações. Esse tipo de intervenção pode causar infecção e hemorragia para mãe e ao recémnascido síndrome de angustia respiratória e maiores proporções de prematuros (Alves, et al., 2021).

Em uma coorte de puérperas atendidas em um hospital da região oeste de Santa Catarina evidenciou que as cesarianas foram maiores em mulheres brancas (Ongaratto, Bomfim \& Rossetto, 2021). Essa característica foi semelhante ao encontrado numa área no município do Rio de Janeiro.

\section{Considerações Finais}

A avaliação do perfil epidemiológico dos nascidos vivos deve fazer parte dos serviços de saúde para que as ações estratégicas sejam direcionadas, com vistas a intervir nos agravos de saúde que levam a situação de prematuridade, a desigualdade do acesso ao pré-natal, e cesarianas sem critério. Infere-se que mesmo com o avanço das políticas públicas os percentuais de prematuridade, Apgar no primeiro minuto e as cesarianas ainda estão acima do preconizado pela Organização Mundial de Saúde.

A análise comparativa entre as áreas de planejamento em saúde leva aos gestores dos serviços locais o conhecimento 
de que o perfil epidemiológico de mulheres e neonatos se comportam de modo diferente. Portanto essa pesquisa contribui para que a secretaria de saúde do referido município possa criar planos de ações de acordo com o real cenário de cada área, direcionando ações resolutivas para melhor assistência à saúde e prevenção de mortalidade materno-infantil.

Conclui-se que para compreender essa situação é necessário realizar estudos mais apurados que investiguem as causas desses agravos em saúde em um território marcado por mosaicos socioeconômicos e políticas iguais para populações distintas. Este estudo traz como limitações o viés da falácia ecológica, visto que, a análise realizada é do agregado e não a exposição e desfecho de cada indivíduo, tipificando uma limitação.

Sugere-se que sejam realizados estudos com o objetivo de analisar ao longo do tempo o perfil de mulheres que cursam com o desfecho cesariana e ou prematuridade do recém-nascido. Estudo de coorte que possa avaliar doenças pré-existentes, antecedentes obstétricos e comportamentos das gestantes.

\section{Referências}

Aldrighi, J. D., Wall, M. L., Souza, S. R. R. K. \& Cancela, F. Z. V. (2016). As experiências das mulheres na gestação em idade materna avançada: revisão integrativa. Rev Esc Enferm USP, 50 (3), 512-521. https://www.scielo.br/pdf/reeusp/v50n3/pt_0080-6234-reeusp-50-03-0512.pdf. 10.1590/S0080623420160000400019

Alencar, N. P. F. C., Castro, J. M., Martins, R. E. C., Azevedo, M. A., Oliveira, T. V. C., Proti, E. S., Araújo, D. A., Arruda, J. S. D., Guerra, C. H. W. \& Costa, W. J. T. (2020). Perfil de nascimentos no estado de Minas Gerais no período de 2013 a 2017. Revista Eletrônica Acervo Enfermagem, 2, 1-7. https://acervomais.com.br/index.php/enfermagem/article/download/2354/1266/. doi:10.25248/REAenf.e2354.2020

Almeida, B. B. P., Morales, J. D. C., Luz, G. S., Rissardo, L. K., Pelloso, S. M. \& Antunes, M. B. (2018). Idade materna e resultados perinatais na gestação de alto risco. Nursing, 21 (247), 2513-2517. https://pesquisa.bvsalud.org/portal/resource/pt/biblio-970954.

Almeida, R. S., Almeida, Y. S., Menezes, M. O., Pinto, K. B. \& Lobo, L. S. (2020). Caracterização da adesão ao pré-natal e desfecho gestacional em Aracaju/SE através da declaração de nascido vivo. Ciências Biológicas e de Saúde Unit, 6 (1), 75-87. https://periodicos.set.edu.br/cadernobiologicas/article/view/7758.

Alves, N. C. C., Feitosa K. M. A., Mendes, M. E. S. \& Caminha, M. F. C. (2017). Complicações na gestação em mulheres com idade maior ou igual a 35 anos. Rev Gaúcha Enferm, 38 (4), 1-8. https://www.scielo.br/pdf/rgenf/v38n4/1983-1447-rgenf-38-04-e2017-0042.pdf. 10.1590/1983-1447.2017.04.2017-0042

Alves, R. S. S., Silva, M. P. B., Leite, A. C., Silva, E. R., Cunha, J. A., Carvalho, M. M., Pereira, B. L., Silva, L. C., Passos, A. D., Morais, M. P. S., Sucupira, K. S. M. B., Sisconetto, A. T., Silva, V. C. R., Apolinário, J. M. S. S., Moura, L. C., Oliveira, A. E. A., Amando, M. A. O., Machado, M. E. M. B. \& Alencar, V. P. (2021). Análise e monitoramento das taxas de cesárea no Brasil segundo a classificação de Robson. Research, Society and Development,10 (6), 1-7. https://rsdjournal.org/index.php/rsd/article/view/15523/13785. doi:10.33448/rsd-v10i6.15523

Anjos, J. C. \& Boing, A. F. (2016). Diferenças regionais e fatores associados ao número de consultas de pré-natal no Brasil: análise do Sistema de Informações sobre Nascidos Vivos em 2013. Rev. bras. epidemiol., 19 (4), 835-850. https://www.scielo.br/j/rbepid/a/fWsgnnQVHNYBSbrYv5ZR8NJ/abstract/?lang=pt. $10.1590 / 1980-5497201600040013$

Barrios, T. V., Souza, L. C., Diaz, C. M. G., Marques, C. T., Carrion, K. T. S., Zamberlan, C. \& Menezes, F. L. (2021). Perfil de cesarianas de uma maternidade do interior do Rio Grande do Sul. Research,Society and Development, 10 (1), 1-8. https://rsdjournal.org/index.php/rsd/article/view/11747/10588. http://dx.doi.org/10.33448/rsd-v10i1.11747

Bulhoes, T. R. B., Alves, J. B., Moreno, C. A., Silva, T. B. \& Dutra, L. P. (2017). Prevalência de Recém-Nascidos Pré-Termo de Mães Adolescentes. Id on Line Rev. Mult. Psic., 11 (39), 84-96. https://idonline.emnuvens.com.br/id/article/view/950.

Cardoso, D. J. S. \& Schumach, B. (2017). Características epidemiológicas das internações neonatais em uma maternidade pública. Rev. enferm. UFPI, 6 (4), 28-32. https://pesquisa.bvsalud.org/portal/resource/pt/bde-33138\#: :text=Resultados\%3A,52\%2C7\%25\%2C\%20respectivamente.

Costa, M. F. S., Gomes Junior, S. C. \& Magluta C. (2018). Análise da distribuição dos nascimentos com marcadores de gravidade em maternidades com unidade de terapia intensiva neonatal no Sistema Único de Saúde. Cad. Saúde Colet., 26 (2), 125-130. https://www.scielo.br/j/cadsc/a/SyDVCwgx5C6nbYBLrwXGDqg/?lang=pt\&format=pdf. doi:10.1590/1414-462X201800020419

Faria, R. \& Santana, P. (2016). Variações espaciais e desigualdades regionais no indicador de mortalidade infantil do estado de Minas Gerais, Brasil. Saude soc., 25 (3), 736-749. https://www.scielo.br/j/sausoc/a/3K6QpcRTdMh68CMPp8tct5k/abstract/?lang=pt. 10.1590/S0104-12902016147609

Ferreira, E. T. M., Moura, N. S., Gomes, M. L. S., Silva, E. G., Guerreiro, M. G. S. \& Oriá, M. O. B. (2019). Características maternas e fatores de risco para pré-eclâmpsia em Gestantes. Rev Rene, 20 (1), 1-7. http://periodicos.ufc.br/rene/article/view/40327/pdf. 10.15253/2175-6783.20192040327

Freitas, P. F. \& Araújo, R. R. (2015). Prematuridade e fatores associados em Santa Catarina, Brasil: análise após alteração do campo idade gestacional na Declaração de Nascidos Vivos. Rev. Bras. Saúde $\quad$ Matern. $\quad$ Infant., 15 309-316. https://www.scielo.br/j/rbsmi/a/xq7RjNbK3WLTM9ZLmGG6w5t/?format=pdf. 10.1590/S1519-38292015000300006

Fundo das Nações Unidas para a Infância. (2019a). 17 objetivos para transformar nosso mundo. https://nacoesunidas.org/pos2015/\#

Fundo das Nações Unidas para a Infância. (2019b). Quase 400 mil bebês nasceram em todo mundo no $1^{\circ}$ dia de 2019 . https://nacoesunidas.org/unicef-quase400-mil-bebes-nasceram-em-todo-mundo-no-1o-dia-de-2019 
Gordis, L. (2010). Epidemiologia (4a ed.). Revinter.

Justino, D. C. P., Lopes, M. S., Santos, C. D. P. \& Andrade, F. B. (2019). Avaliação histórica das políticas públicas de saúde infantil no Brasil: revisão integrativa. Revista Ciência Plural. 5 (1), 71-88. https://periodicos.ufrn.br/rcp/article/view/17946. 10.21680/2446-7286.2019v5n1ID17946

Madeiro, A., Rufino, A. C. \& Santos, A. O. (2017). Partos cesáreos no Piauí: tendência e fatores associados no período 2000-2011. Epidemiol. Serv. Saude, 26 (1), 81-90. https://www.scielo.br/j/ress/a/HpGL9Qbh4gHX3bVRjZS9nRL/?lang=pt. 10.5123/S1679-49742017000100009

Maia, V. K. V., Lima, E. F. A., Leite, F. M. C., Sousa, A. I. \& Primo, C. C. (2017). Avaliação dos indicadores de processo do Programa de Humanização no Pré-natal e Nascimento e da Rede Cegonha. J. res.: fundam. care. Online, 9 (4), 1055-1060. https://pesquisa.bvsalud.org/portal/resource/pt/biblio-908511. 10.9789/2175-5361.2017.v9i4.1055-10

Marques, L. J. P., Oliveira, C. M. \& Bonfim, C. V. (2016). Avaliação da completude e da concordância das variáveis dos Sistemas de Informações sobre Nascidos Vivos e sobre Mortalidade no Recife-PE, 2010-2012. Epidemiol. Serv. Saúde, 25 (4), 849-854. https://www.scielo.br/j/ress/a/9RLXqjMF5DTZ9nD3bSHGHVG/abstract/?lang=pt. 10.5123/S1679-49742016000400019

Muniz, E. B., Vasconcelos, B. B., Pereira, N. A., Frota, R. G., Moraes, C. E. B., \& Oliveira, M. A. S. (2016). Análise do boletim de Apgar em dados do Sistema de Informação sobre Nascidos Vivos registrados em um hospital do interior do estado do Ceará, Brasil. Rev Med Saude Brasilia, 5 (2), 182-191. https://portalrevistas.ucb.br/index.php/rmsbr/article/view/6677.

Nunes, F. B. B. F., Prudêncio, P.S., Carvalho, J. F. S. \& Mamede, F. V. (2016). Incompletude de informação de nascidos vivos em São Luís/MA no ano de 2012. J. res.: fundam. care. Online, 8 (1), 3705-3713. de https://pesquisa.bvsalud.org/portal/resource/pt/lil-776723. 10.9789/2175-5361.2016.v8i1.3705-3713

Oliveira, M. M., Andrade, S. S. C. A., Dimech, G. S., Oliveira, J. C. G., Malta, D. C., Neto, D. L. R. \& Moura, L. (2015). Avaliação do Sistema de Informações sobre Nascidos Vivos. Brasil, 2006 a 2010. Epidemiol. Serv. Saúde, 24 (4), 629-640. https://www.scielo.br/j/ress/a/bRpxYCk4zyh4NwK84yyTKFj/abstract/?lang=pt. 10.5123/S1679-49742015000400005

Ongaratto, F., Bomfim, S. B. \& Rossetto, M. (2021). Características sociodemográficas e clínicas de uma coorte de puérperas em um hospital de Santa Catarina. Research, Society and Development, 10 (1), 1-12. https://rsdjournal.org/index.php/rsd/article/view/11288/10595. 10.33448/rsd-v10i1.11288

Organização Pan-Americana da Saúde. (2019). Os Objetivos de Desenvolvimento do Milênio e a Agenda Pós 2015 . https://www.paho.org/bireme/index.phpoption=com_content\&view=article\&id=301:os-objetivos-de-desenvolvimento-do-milenio-e-a-agenda-pos2015\&Itemid=183\&lang=pt

Pedraza, D. F. \& Cristóvão, F. S. (2015). Fatores associados ao peso ao nascer: estudo populacional com base nas declarações de nascidos vivos. Arquivos de Ciências da Saúde, 22 (4), 92-99. https://www.cienciasdasaude.famerp.br/index.php/racs/article/view/105. 10.17696/2318-3691.22.4.2015.105

Pereira, M. U. L., Lamy Filho, F., Anunciação, P. S., Lamy, Z. C., Gonçalves, L. L. M. G. \& Madeira, H. G. R. (2017). Óbitos neonatais no município de São Luís: causas básicas e fatores associados ao óbito neonatal precoce. Rev Pesq Saúde, 18 (1), 18-23. http://www.periodicoseletronicos.ufma.br/index.php/revistahuufma/article/view/7874.

Pereira, R. S. V \& Mudjalieb, A. A. (2016). O desafio da mudança do modelo assistencial ao parto e nascimento nas Maternidades Prioritárias do estado do Rio de Janeiro. Divulg. saúde debate, (54), 57-63. https://pesquisa.bvsalud.org/portal/resource/pt/biblio-996857.

Pinto Junior, E. P., Luz, L. A., Guimarães, M. A. P., Tavares, L. T., Brito, T. R. S. \& Souza, G. D. F. (2017). Prevalência e fatores associados às anomalias congênitas em recém-nascidos. Rev Bras Promoç Saúde, 30 (3), 1-9. https://periodicos.unifor.br/RBPS/article/view/6467. 10.5020/18061230.2017.6467

Rattner, D. \& Moura, E. C. (2016). Nascimentos no Brasil: associação do tipo de parto com variáveis temporais e sociodemográficas. Rev. Bras. Saude Mater. Infant., 16 (1), 39-47. https://www.scielo.br/j/rbsmi/a/pRg8tcZQgDLBHFdz8bLsWBr/abstract/?lang=pt. 10.1590/1806-93042016000100005

Renner, F. W., Garcia, E. L., Renner, J. D. P., Costa, B. P., Figueira, F. P., Ebert, J. P., Nascimento, L. S., Ferrari, L., Grossi, M. \& França, V. T. (2015). Perfil epidemiológico das puérperas e dos recém-nascidos atendidos na maternidade de um hospital de referência do interior do Rio Grande do Sul no primeiro semestre de 2014. Boletim Científico de Pediatria, 4 (2), 27-32. https://www.sprs.com.br/sprs2013/bancoimg/160107101642bcped_v4_n2_a2.pdf.

Rio de Janeiro. Secretaria Estadual de Saúde. (2017). Plano de Ação do Programa Rede Cegonha no SUS - RJ: manual técnico de ação integrada a saúde da gestante, puérpera e recém nascido [manual]. SMS/RJ.

Silva, A. M. (2016). Atenção ao pré-natal, parto e pós-parto na perspectiva de mulheres atendidas na Rede Cegonha. Tese de doutorado, Escola de Enfermagem Anna Nery, Rio de Janeiro, RJ, Brasil.

Silva, E. P., Lima, R. T. \& Osório, M. M. (2016). Impacto de estratégias educacionais no pré-natal de baixo risco: revisão sistemática de ensaios clínicos randomizados. Ciênc. saúde colet., 21(9),2935-2948. https://www.scielo.br/j/csc/a/bVt77Sfv5YWZvHjrpKkTQpg/abstract/?lang=pt. 10.1590/141381232015219.01602015

Teixeira, G. A., Carvalho, J. B. L., Rocha, B. G., Pereira, S. A. \& Enders B. C. (2018). Perfil de mães e o desfecho do nascimento prematuro ou a termo. Cogitare Enferm., 23 (1), 1-10. https://revistas.ufpr.br/cogitare/article/view/51409. 10.5380/ce.v23i1.51409

Texeira, G. A., Costa, F. M. L., Mata, M. S., Carvalho, J. B. L., Souza, N. L., Silva, R. A. R. (2016). Fatores de risco para a mortalidade neonatal na primeira semana de vida. J. res.: fundam. care. Online, 8 (1), 4036-4046. https://pesquisa.bvsalud.org/portal/resource/pt/lil-776214. 10.9789/2175-5361.2016.v8i1.4036-4046

Timm, I. C., Rodrigues, A. M., Valverde, A. M. \& Ribeiro, C. B. (2019). Avaliação da qualidade da assistência pré-natal em uma unidade básica de saúde do município de Pelotas - RS. Braz. J. Hea. Rev., 2 (4), 3729-3735. https://www.brazilianjournals.com/index.php/BJHR/article/viewFile/2646/2654. $10.34119 /$ bjhrv2n4-134

Vieira, I. B., Oliveira, P. S. D., Sanches, G. L. G., Miranda, S. V. C., Leão, H. M. \& Ribeiro, N. G. (2017). Fatores de riscos e implicações da gravidez na adolescência: uma revisão integrativa de literatura. Revista Eletrônica Acervo Saúde.7, 544-549. https://www.acervosaude.com.br/doc/REAS9.pdf. 